\title{
Location and Shape Reconstruction of 2D Dielectric Objects by Means of a Closed-Form Method: Preliminary Experimental Results
}

\author{
Gian Luigi Gragnani and Maurizio Diaz Mendez \\ Diten, University of Genoa, Via Opera Pia 11A, 16145 Genoa, Italy \\ Correspondence should be addressed to Gian Luigi Gragnani, gianluigi.gragnani@unige.it
}

Received 30 March 2012; Accepted 31 May 2012

Academic Editor: Andrea Randazzo

Copyright ( 2012 G. L. Gragnani and M. Diaz Mendez. This is an open access article distributed under the Creative Commons Attribution License, which permits unrestricted use, distribution, and reproduction in any medium, provided the original work is properly cited.

\begin{abstract}
An analytical approach to location and shape reconstruction of dielectric scatterers, that was recently proposed, is tested against experimental data. Since the cross-sections of the scatterers do not depend on the $z$ coordinate, a 2D problem can be formulated. A closed-form singular value decomposition of the scattering integral operator is derived and is used to determine the radiating components of the equivalent source density. This is a preliminary step toward a more complete solution, which will take into account the incident field inside the investigation domain in order to provide the dielectric features of the scatterer and also the nonradiating sources. Reconstructions of the equivalent sources, performed on some scattering data belonging to the Fresnel database, show the capabilities of the method and, thanks to the closed-form solution, results are obtained in a very short computation time.
\end{abstract}

\section{Introduction}

In the last decades, inverse electromagnetic scattering and near field imaging have been widely studied research topics [1]. Actually, electromagnetic imaging [2] is a very promising technique in many practical application fields. The more consolidated applications perhaps are those related to the use of ground penetrating radar, but a lot more are at present used or in advanced state of development. For example, it has been suggested that microwave imaging could be successfully used as a diagnostic technique in several areas, including civil and industrial engineering [3-5], nondestructive testing and evaluation [6-9], geophysical prospecting [10], and biomedical engineering [11-14]. Microwave imaging has also been proposed as a useful tool in wood industry [15]. Also, sophisticated techniques for plasma diagnostic can be based on microwave imaging [16]. Furthermore, inverse electromagnetic scattering can provide insights for approaching other problems of great interest nowadays, for example, plasmonic cloaking and the synthesis of metamaterials [17].

One of the aim of an inverse electromagnetic-scattering imaging system is to find the actual position of a dielectric object inside a bounded space region, as well as its shape. The techniques applied are based on the use of a known incident field illuminating the space region containing the object. By suitably measuring the scattered field, a solution to the problem can be derived.

The main difficulties with solving an inverse electromagnetic-scattering problem result from its nonlinearity and instability. To overcome these negative features, in the scientific literature, several regularization techniques have been proposed. Furthermore, the a priori knowledge usually available could be included in the formulation of the resolving algorithm. The problem could be recast as a global nonlinear optimization problem, and stochastic, as well as deterministic approaches can be used to achieve a solution. The interested reader is referred, for example, to [18-22]. 
Another common technique to overcome nonlinearity is based on the transformation of the original problem into an inverse source one, by replacing the scatterer with an equivalent (induced) source. Although the equivalent source density linearizes the inverse scattering equation, the transformation of the inverse scattering problem into an inverse source one involves the solution of an integral equation of the first kind. Hence, the resulting operator is ill-posed, and its solution is usually unstable and nonunique [23]. In this particular case, nonuniqueness is due to the socalled nonradiating sources, that do not radiate outside their domain of definition and hence belong to the null space of the scattering operator [24].

In the present work, an approach to the inverse source problem that allows to clearly reformulate it in terms of both the radiating and nonradiating parts of the induced sources is used. Through a singular value decomposition (SVD) of the scattering operator, a closed-form solution to the radiating sources is reached. By using such a solution, an expression for the reconstructed scattered field inside the dielectric object can also be obtained in closed form.

While the theory that is at the base of this work was already presented and tested by numerical simulations [25, 26], the present paper is devoted to the reconstruction of the scattering properties from experimental data. Testing inversion algorithms against experimental data is a very important topic to deal with. Actually, since inverse scattering problems are ill-posed, their solutions are not as easy to be obtained as in the case of direct problems, and the risk in not validating an imaging algorithm with real world data is that the simulations could not take into account all the parameters involved in reality. Simulations can be made more and more complex, thanks to the availability of computers with continuously increasing power, however researchers must validate the model with experiments, refine it, and simulate and test again, and iteratively repeat the process until the model matches the physical data. Fortunately, in the last decade, some research groups have dealt with this lack of experimental data by performing measurements on a controlled environment and by making available the results to the inverse scattering community. The first available data were those of the so-called Ipswich database [27-30]. A larger collection of data, on both metallic and dielectric scatterers, is now available thanks to the efforts of the group at the Institut Fresnel, Marseille, France [31, 32]. In the present work, some of the data of the Fresnel database are used to test the inversion method. Since one of the interesting features of the Fresnel database is that data are provided over a wide band frequency range, also some comparisons about the behavior of the method at different frequencies have been carried out.

Although the present work is at an early stage and only some features of the theoretical model have been checked against the experimental data, the method seems to perform well, and its capabilities to reconstruct both the location and the shape of the induced sources are demonstrated. Furthermore, thanks to the closed-form formulation of the solution, results are available in a very short time that can allow for a quasi real-time imaging system.

\section{Mathematical Background}

In this section, the closed-form solution to the inverse scattering problem is briefly resumed. For more details the reader is referred to $[25,26]$.

2.1. The Electromagnetic Scattering. Let us consider an inhomogeneous object whose dielectric parameters are unknown, and which is irradiated by an electromagnetic field produced by a known source. The presence of the object leads to a total electromagnetic field that is different from the one that the same source would radiate in a free-space environment. In particular, the scattered field due to the object can be modeled by means of the volume equivalence theorem [33], that allows one to replace the dielectric object with an induced (or equivalent) source density, radiating in free space. So the object acts as a secondary source of radiated electromagnetic field. In three dimensions, the scattered field vector $\mathbf{E}_{s}$ can be expressed as

$$
\begin{aligned}
\mathbf{E}_{s}(\mathbf{r})= & \mathbf{E}_{\mathrm{tot}}(\mathbf{r})-\mathbf{E}_{\mathrm{inc}}(\mathbf{r}) \\
= & \omega^{2} \mu \varepsilon \iiint \mathbf{P}_{\mathrm{eq}}^{\mathrm{TOT}}\left(\mathbf{r}^{\prime}\right) \mathcal{g}\left(\left|\mathbf{r}-\mathbf{r}^{\prime}\right|\right) \mathrm{d} \mathbf{r}^{\prime} \\
& +\nabla \nabla \cdot \iiint \mathbf{P}_{\mathrm{eq}}^{\mathrm{TOT}}\left(\mathbf{r}^{\prime}\right) \mathcal{g}\left(\left|\mathbf{r}-\mathbf{r}^{\prime}\right|\right) \mathrm{d} \mathbf{r}^{\prime},
\end{aligned}
$$

where

(i) $\mathbf{E}_{\text {tot }}$ is the total electrical field vector;

(ii) $\mathbf{E}_{\text {inc }}$ is the incident field vector, that is the known field that would be radiated without the object;

(iii) $\mathbf{r}=\mathbf{r}(\rho, \vartheta, z)$ is the position vector of the observer;

(iv) $\mathbf{r}^{\prime}=\mathbf{r}^{\prime}\left(\rho^{\prime}, \vartheta^{\prime}, z^{\prime}\right)$ is the position vector of any point of the scattering domain;

(v) $g\left(\left|\mathbf{r}-\mathbf{r}^{\prime}\right|\right)$ is the Green's function for the electromagnetic radiation in free space;

(vi) $\varepsilon$ is the dielectric permittivity of the propagation medium;

(vii) $\mu$ is the magnetic permeability of the propagation medium;

(viii) $\mathbf{P}_{\mathrm{eq}}^{\mathrm{TOT}}$ is a polarization field that acts as an equivalent source for the scattered field.

Within the assumption of dealing with nonmagnetic media (i.e., $\mu=\mu_{0}$ both inside and outside the scatterers, $\mu_{0}$ being the permeability in vacuo), we have [34]

$$
\mathbf{P}_{\mathrm{eq}}^{\mathrm{TOT}}(\mathbf{r})=\tau(\mathbf{r}) \mathbf{E}_{\mathrm{tot}}(\mathbf{r}),
$$

where

(i) $\tau(\mathbf{r})=\left(\varepsilon_{r}(\mathbf{r})-1\right)$ is called scattering potential and $\varepsilon_{r}(\mathbf{r})$ is the complex relative dielectric permittivity of the scatterer, with respect to the background permittivity $\varepsilon$. 


\subsection{D TM Model}

The problem is simplified into a two-dimensional and scalar one, when

(1) the scatterers are infinitely extended along $z$;

(2) the scattering potential does not depend on $z$;

(3) the incident electric field does not depend on $z$, is $z$ directed, and propagates in a transverse direction (TM illumination).

In this case also the scattered field is directed along $z$ and only the cross section with respect to $z$ must be considered. The integral equation modeling the inverse scattering problem becomes [35]

$$
\begin{aligned}
E_{s}(\boldsymbol{\rho}) & =E_{\mathrm{tot}}(\boldsymbol{\rho})-E_{\mathrm{inc}}(\boldsymbol{\rho}) \\
& =-j \frac{k^{2}}{4} \iint_{D_{\mathrm{inv}}} \tau\left(\boldsymbol{\rho}^{\prime}\right) E_{\mathrm{tot}}\left(\boldsymbol{\rho}^{\prime}\right) H_{0}^{(2)}\left(k\left|\boldsymbol{\rho}-\boldsymbol{\rho}^{\prime}\right|\right) d \boldsymbol{\rho}^{\prime},
\end{aligned}
$$

where, in a generic cross section of the object:

(i) $E_{\text {tot }}$ is the $z$ component of the total electrical field;

(ii) $E_{\text {inc }}$ is the $z$ component of the incident field;

(iii) $\boldsymbol{\rho}=\boldsymbol{\rho}(\rho, \vartheta)$ is the position vector of the observer;

(iv) $\boldsymbol{\rho}^{\prime}=\boldsymbol{\rho}^{\prime}\left(\rho^{\prime}, \vartheta^{\prime}\right)$ is the position vector of any point of the investigation domain $D_{\text {inv }}$;

(v) $H_{0}^{(2)}\left(k\left|\rho-\rho^{\prime}\right|\right)$ is the second-kind and zeroth-order Hankel function;

(vi) $k=\omega \sqrt{\mu \varepsilon}$ is the wave number.

In the paper, it is assumed that scatterers are placed inside a circular region of radius $R$, along its circumference the scattered electromagnetic field is measured. The circular region defines the geometry of the problem and is called the domain of investigation $D_{\text {inv }}$, while the circle of sensors is called the domain of observation $D_{\text {obs }}$.

As it was already mentioned, the inverse problem given by (3) is nonlinear because such an equation contains the product of the total field $E_{\text {tot }}\left(\rho^{\prime}\right)$ and the scattering potential $\tau\left(\rho^{\prime}\right)$, both of which are unknown.

The problem can be linearized by using the equivalent source $P_{\mathrm{eq}}^{\mathrm{TOT}}$. It must be noticed that since in this way an equivalent inverse source problem is faced instead of an inverse scattering one, both the radiating and nonradiating contributions, $P_{\mathrm{eq}}$ and $P_{\mathrm{eq}}^{\mathrm{NR}}$, of the source should be accounted for. Hence $P_{\mathrm{eq}}^{\mathrm{TOT}}$ can be rewritten as

$$
P_{\mathrm{eq}}^{\mathrm{TOT}}\left(\boldsymbol{\rho}^{\prime}\right)=\tau\left(\boldsymbol{\rho}^{\prime}\right) E_{\mathrm{tot}}\left(\boldsymbol{\rho}^{\prime}\right)=P_{\mathrm{eq}}\left(\boldsymbol{\rho}^{\prime}\right)+P_{\mathrm{eq}}^{\mathrm{NR}}\left(\boldsymbol{\rho}^{\prime}\right), \quad \boldsymbol{\rho}^{\prime} \in \mathrm{D}_{\mathrm{inv}}
$$

and the inverse problem can be formulated as

$$
E_{s}(\boldsymbol{\rho})=-j \frac{k^{2}}{4} \iint_{D_{\mathrm{inv}}} P_{\mathrm{eq}}^{\mathrm{TOT}}\left(\boldsymbol{\rho}^{\prime}\right) H_{0}^{(2)}\left(k\left|\boldsymbol{\rho}-\boldsymbol{\rho}^{\prime}\right|\right) d r^{\prime} .
$$

As compared to other nonlinear methods (see, e.g., [12, 13]) where there is no separation between radiating and nonradiating sources, in the present approach it is clearly identified which part of the reconstruction can be obtained by measurements. Further improvement can then only be obtained by means of the nonradiating sources and by using the constraint that (3) must hold also inside the investigation domain.

2.3. Radiating Source. The radiating part $P_{\mathrm{eq}}$ of the induced source can be computed after the derivation of the singular value decomposition of (5) and the definition of its singular system $\left\{w_{\mu}, \varphi_{\mu} ; \sigma_{\mu}\right\}$. Detailed mathematical derivations can be found in [26]. In the present section, only the main results of the method are recalled.

For a lossless propagation medium, the singular system $\left\{w_{\mu}, \varphi_{\mu} ; \sigma_{\mu}\right\}$ of $(5)$ is

$$
w_{\mu}(9)=\frac{1}{\sqrt{2 \pi R}} e^{j \mu \vartheta},
$$

$$
\begin{aligned}
& \varphi_{\mu}(\rho, \vartheta) \\
& =j \frac{1}{\sqrt{2 \pi}} \frac{H_{\mu}^{(2)}(k R) J_{\mu}(k \rho)}{\left|H_{\mu}^{(2)}(k R)\right| \sqrt{\left(R^{2} / 2\right)\left[J_{\mu}^{2}(k R)-J_{\mu+1}(k R) J_{\mu-1}(k R)\right]}} \\
& \quad \times e^{j \mu \vartheta}, \\
& \quad \sigma_{\mu}=\frac{k^{2} \pi \sqrt{R}}{2}\left|H_{\mu}^{(2)}(k R)\right| \\
& \quad \times \sqrt{\frac{R^{2}}{2}\left[J_{\mu}^{2}(k R)-J_{\mu+1}(k R) J_{\mu-1}(k R)\right]},
\end{aligned}
$$

where

(i) the functions $w_{\mu}(9)$ are the left singular functions of the decomposition;

(ii) the functions $\varphi_{\mu}(\rho, 9)$ are the right singular functions of the decomposition;

(iii) the numbers $\sigma_{\mu}$ are the singular values,

and where $H_{\mu}^{(2)}$ and $J_{\mu}$ are the second-kind Hankel function of order $\mu$ and the first-kind Bessel function of order $\mu$, respectively.

A solution to the radiating source contribution $P_{\mathrm{eq}}$ in term of singular system can be written as [36]

$$
P_{\mathrm{eq}}(\boldsymbol{\rho})=\sum_{\mu=1}^{\infty} \frac{1}{\sigma_{\mu}} \varphi_{\mu} \int_{0}^{2 \pi} E_{\mathrm{scatt}} w_{\mu}^{*} R d \vartheta
$$


where the asterisk means complex conjugate. When the values given by (6) are substituted into (7), the following expression for the equivalent source is obtained:

$$
\begin{aligned}
& P_{\text {eq }}(\rho, \vartheta) \\
& =\frac{2 j}{k^{2} \pi} \\
& \quad \times \sum_{\mu=-\infty}^{+\infty} c_{\mu} \frac{H_{\mu}^{(1)}(k R)}{\left|H_{\mu}^{(2)}(k R)\right|^{2}\left(R^{2} / 2\right)\left[J_{\mu}^{2}(k R)-J_{\mu+1}(k R) J_{\mu-1}(k R)\right]} \\
& \quad \times J_{\mu}(k \rho) e^{j \mu \vartheta},
\end{aligned}
$$

where $c_{\mu}$ stand for the Fourier coefficients of $E_{s}(R, \vartheta)$ with respect to $\vartheta[26]$.

Since $P_{\text {eq }}$ depends on the singular values of the scattering operator, care must be exercised in deriving an expression for the reconstructed radiating source. Actually, the nature of the involved integral equation is such that, in every case, after a certain index, the singular values sharply decrease and tend to zero. These small singular values increase the instability of the solution. In order to minimize such instability, only the singular values above a suitable threshold should be taken into account in the solution.

As for the nonradiating sources $P_{\mathrm{eq}}^{\mathrm{NR}}$, since they are not measurable, they are in general difficult to be reconstructed. In principle, their contributions could be found out by taking into account that they must be orthogonal to the radiating ones and do not radiate outside the domain of investigation, and by using some priors about the scatters. Some, works trying to deal in a formal way with $P_{\mathrm{eq}}^{\mathrm{NR}}$ (see, e.g., $[37,38]$ ) and, in particular, the method presented in [39] fits very well with the present closed-form approach. All these approaches, however, are computationally very expensive. In the most of the imaging systems, nonradiating currents are put to zero. This is a common assumption in the solution of this kind of inverse problems, and this is the line followed in the present paper, in order to have results in short time. Since the nonradiating sources contribute to the higher spatial frequencies, the price to pay for assuming $P_{\mathrm{eq}}^{\mathrm{NR}}$ negligible is usually a smoothing effect in the reconstruction, but the solution is anyway good enough for many purposes in electromagnetic imaging.

2.4. Scattered Field and Scattering Potential. By using the expression given by (8), approximate scattered fields both inside and outside the scatterer can be analytically obtained. Again, the approximation in deriving the scattered fields stands in using only radiating contribution of the sources, that is:

$$
E_{s}(\boldsymbol{\rho}) \approx-j \frac{k^{2}}{4} \int_{D_{\mathrm{inv}}} P_{\mathrm{eq}}\left(\boldsymbol{\rho}^{\prime}\right) H_{0}^{(2)}\left(k\left|\boldsymbol{\rho}-\boldsymbol{\rho}^{\prime}\right|\right) d \boldsymbol{\rho}^{\prime} .
$$

And the scattering potential becomes

$$
\begin{aligned}
\tau(\boldsymbol{\rho}) & \approx \frac{P_{\mathrm{eq}}(\boldsymbol{\rho})}{E_{\mathrm{tot}}(\boldsymbol{\rho})} \\
& \approx \frac{P_{\mathrm{eq}}(\boldsymbol{\rho})}{E_{\mathrm{inc}}(\boldsymbol{\rho})-j\left(k^{2} / 4\right) \int_{D_{\mathrm{inv}}} P_{\mathrm{eq}}\left(\boldsymbol{\rho}^{\prime}\right) H_{0}^{(2)}\left(k\left|\boldsymbol{\rho}-\boldsymbol{\rho}^{\prime}\right|\right) d \boldsymbol{\rho}^{\prime}} .
\end{aligned}
$$

Also for these results, details about the closed-form derivation are given in $[25,26]$. It should be however anticipated that, since the Fresnel database does not provide the values of the incident field inside the investigation domain, a direct implementation of (10) by using the experimental data is not possible. In some cases, the incident field inside $D_{\text {inv }}$ has been inferred from the values in the measurement domain. However, in the present work, we have preferred to focus the attention on the reconstruction of $P_{\text {eq }}$, that is, anyway the first step of any inverse scattering process. It should be nevertheless noticed that reconstructing the scattering potential, not only can provide quantitative information, but also the qualitative information about the location and the shape of the scatterers is usually better that the one provided by the radiating equivalent source. Hence, attempting to reconstruct $\tau$, by using the full procedures proposed in $[26$, 39 ], will be the core of future works involving experimental data.

\section{Experimental Results}

The method was tested against three sets of experimental data involving dielectric scatterers. In particular, the dielectric targets of the first Fresnel dataset [31] were considered, as well as the "FoamDielExt" of the second dataset [40]. Furthermore, although the method was not originally conceived to deal with conducting objects, a test was also performed by using the data of the U-shaped metallic scatterer present in the first dataset.

It should be pointed out that the present theory assumes that data are available over the full circumference. Instead, in any dataset, scattering field measures were taken over an arc of fixed radius with an angular span of only 240 degrees, whereas no data were available in the remaining 120 degrees. Hence, in order to compute the coefficients $c_{\mu}$ appearing in (8), scattering data were continued with zeros. Although this approach is not the best choice and could lead to some overshoot in the Fourier coefficients, it is the simplest one and proved sufficient to achieve reliable results in any testing case.

The tests were focused on the reconstruction of the radiating equivalent source, and, in particular, three parameters involved in the process were considered: the number of singular values used in computing $P_{\mathrm{eq}}$, the operating frequency $f$, and the direction of arrival of the incident field $\vartheta_{v}$. Furthermore, the capabilities of the method of dealing with multiview and multifrequency data were checked against some different strategies of combining the results.

Results are shown by means of normalized maps of the reconstructed domain. On the same maps also the shape and 
the location of the scattering object are shown, in order to give an immediate feeling about the fairness of the result.

Although both [31, 40] suggest that the investigation domains can be restricted to a small square around a scatterer, this information is not used in the present work, and the whole circle bounded by the measurement circle is considered to belong to the investigaed domain. However, to increase the legibility of the maps, only the square area of $30 \times 30 \mathrm{~cm}^{2}$ around the scatter is shown. Anyway, it should be stressed that the value of the reconstructed equivalent source outside this area is negligible in any case.

Thanks to the closed-form formulation of the solution, all the results were obtained in a very short computation time. In particular, on a single-core class PC, a single view reconstruction takes about $2 s$. Since no optimization of the code was performed, it is expected that this figure can become of magnitude smaller on a dedicated hardware with optimized code, hence allowing for a quasi real-time imaging system.

Since reconstructions are evaluated from the data collected by a single experiment, only a partial information is available at each view. Furthermore, inspections at different frequencies also provide different information. To enhance the overall result, three different possibilities were considered: summing the partial results over the different views at a fixed frequency, summing over the frequencies at a fixed view, and summing over both frequencies and views. These approaches are summarized by the expressions in (11), where the quantities $P_{F}, P_{V}$, and $\mathcal{P}_{V F}$ are defined as

$$
\begin{gathered}
P_{F}=\sum_{v=1}^{N_{v}}\left|\widetilde{P}_{\mathrm{eq}}\left(f, \vartheta_{v}\right)\right|, \quad P_{V}=\sum_{f=1}^{N_{f}}\left|\widetilde{P}_{\mathrm{eq}}\left(f, \vartheta_{v}\right)\right|, \\
\mathcal{P}_{V F}=\sum_{f=1}^{N_{f}} P_{f}=\sum_{v=1}^{N_{v}} P_{v},
\end{gathered}
$$

and where the quantity $\left|\widetilde{P}_{\mathrm{eq}}\left(f, \vartheta_{v}\right)\right|$ is the map of the amplitude of the reconstructed equivalent source at frequency $f$ and at view $\vartheta_{v}$.

3.1. Single Circular Cylinder. The first results are about the inversion of the scattering data produced by a singular dielectric cylinder with a circular cross section of radius $a=$ $15 \mathrm{~mm}$. According to [31], the relative dielectric constant is $\varepsilon_{r}=3 \pm 0.3$ and the center of the object is shifted, in one direction, of about $30 \mathrm{~mm}$, with respect to the origin of the axes. Data are collected over a circle of radius $R=0.76 \mathrm{~m}$ and 48 samples per frequency are available.

A single-view, single-frequency reconstruction is shown in Figure 1. It was obtained at $\vartheta_{v}=0$ and $f=8 \mathrm{GHz}$, and using 8 singular values. The map clearly shows a radiating source in proximity of the true-scattering object, whose maximum value is centered at the side which gave the most significant contribution to the scattered field. Reconstructions obtained by using multiview and multiviewmultifrequency gave encouraging results. In particular, in Figure 2 the quantity $P_{V}$ computed at $f=8 \mathrm{GHz}$ is shown. It can be seen that the circular shape of the object

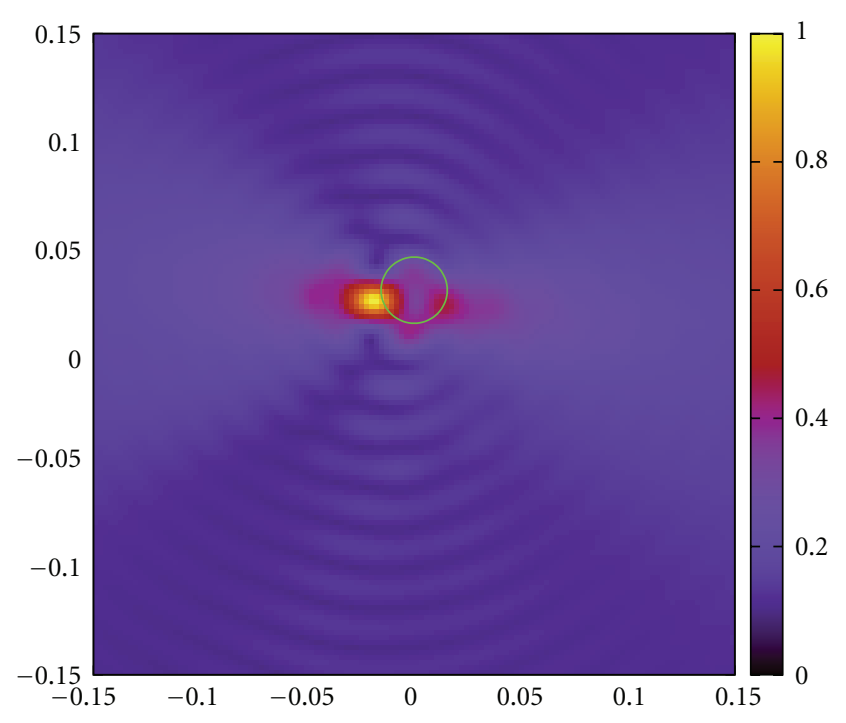

FIGURE 1: Reconstruction of the radiating source for a single dielectric cylinder. Single view $\vartheta_{v}=0$ and single frequency $f=$ $8 \mathrm{GHz} .8$ singular values were used.

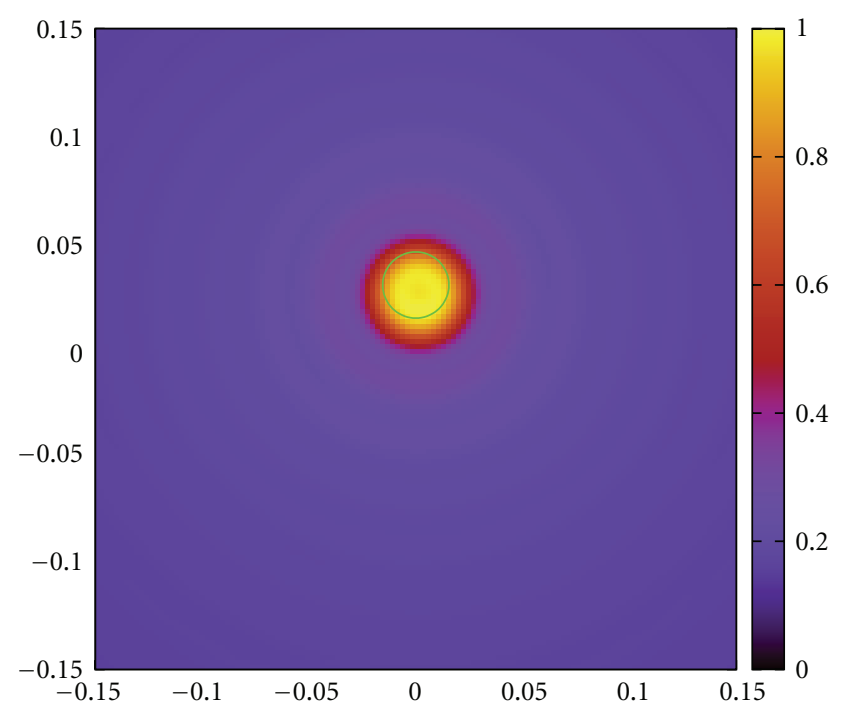

Figure 2: Reconstruction of a single dielectric cylinder. Multiview; map of the $P_{F}$ values computed at $f=8 \mathrm{GHz}$.

is clearly recognized, and also the maximum values of the reconstruction are contained inside the boundary of the true object.

The reconstruction using the multifrequency-multiview approach provided the result shown in Figure 3. Also in this case, the object is clearly reconstructed and, in comparison with Figure 2, the background is much more flat.

3.2. Pair of Circular Cylinders. This configuration was made by a couple of twin dielectric cylinders whose centers are spaced by $90 \mathrm{~mm}$ [31]. The dimensions of the cross-section and the dielectric constant of both cylinders were the same 


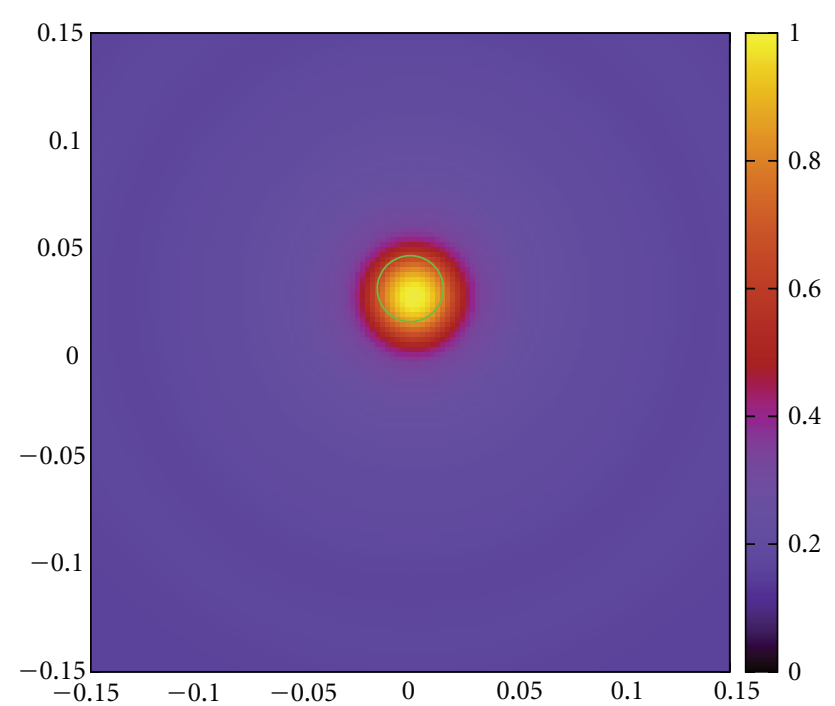

Figure 3: Reconstruction of a single dielectric cylinder. Multiviewmultifrequency. Map of the $\mathcal{P}_{V F}$ values.

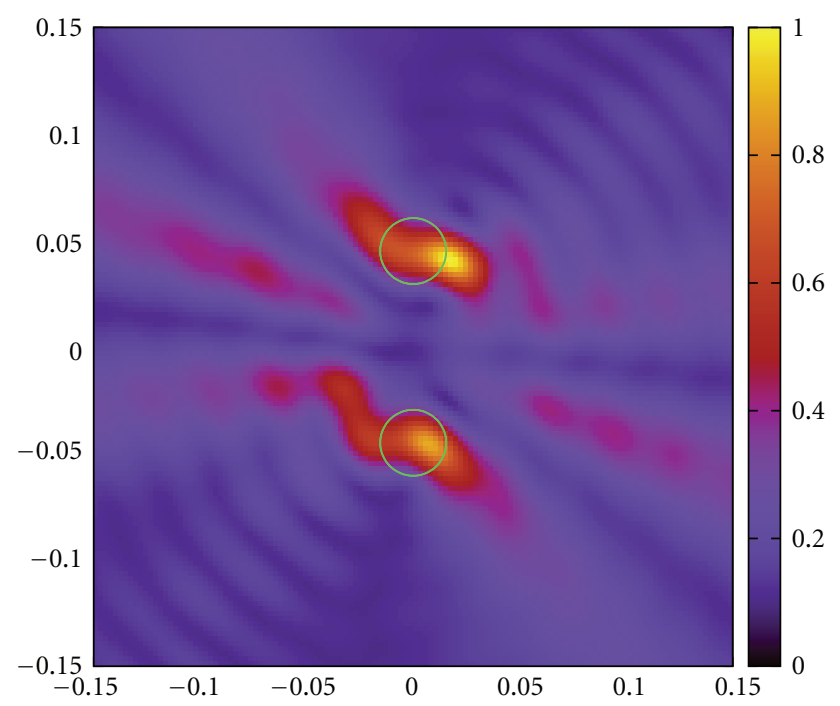

FIGURE 4: Reconstruction of the radiating source for a couple of twin dielectric cylinders. Single view $\vartheta_{v}=0$ and single frequency $f=8 \mathrm{GHz} .8$ singular values were used.

of the cylinder of the previous case, as well as they were the radius of the measurement region and the number of samples.

Single view reconstructions, at each frequency, clearly show the presence of two distinct objects. As an example, in Figure 4 the reconstruction at $\vartheta_{v}=0$ and $f=8 \mathrm{GHz}$ is shown. As in the previous example, 8 singular values were used. In Figure 5, instead, the multifrequency result, for the same illuminating angle $\vartheta_{v}=0$, is shown. With respect to Figure 4, the object is better located and the background appears much more regular. However, also two spurious "scattering queues" are evident.

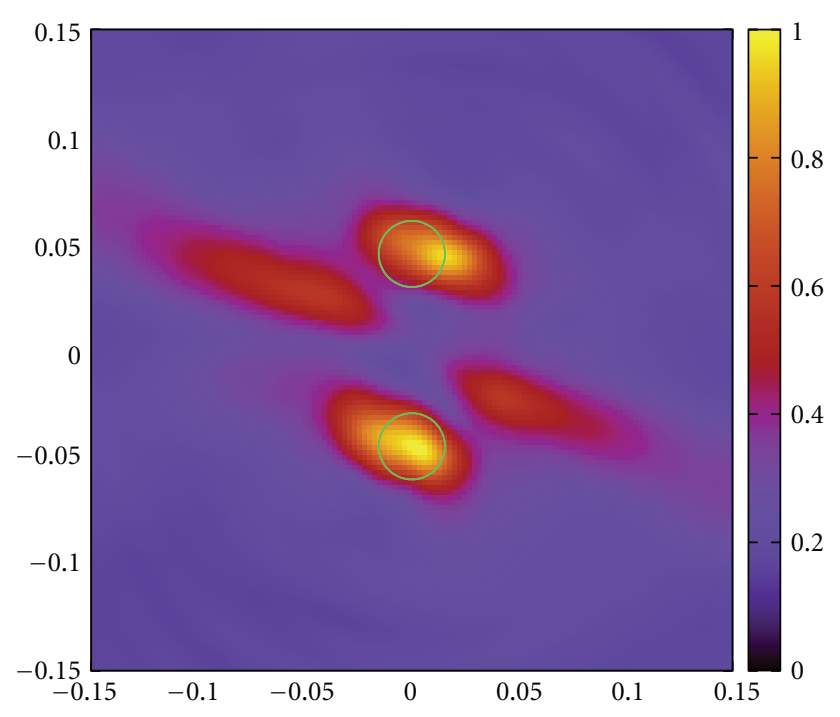

FIGURE 5: Reconstruction of a couple of twin dielectric cylinders. Multifrequency. Map of the $P_{V}$ values computed at $\vartheta_{v}=0$.

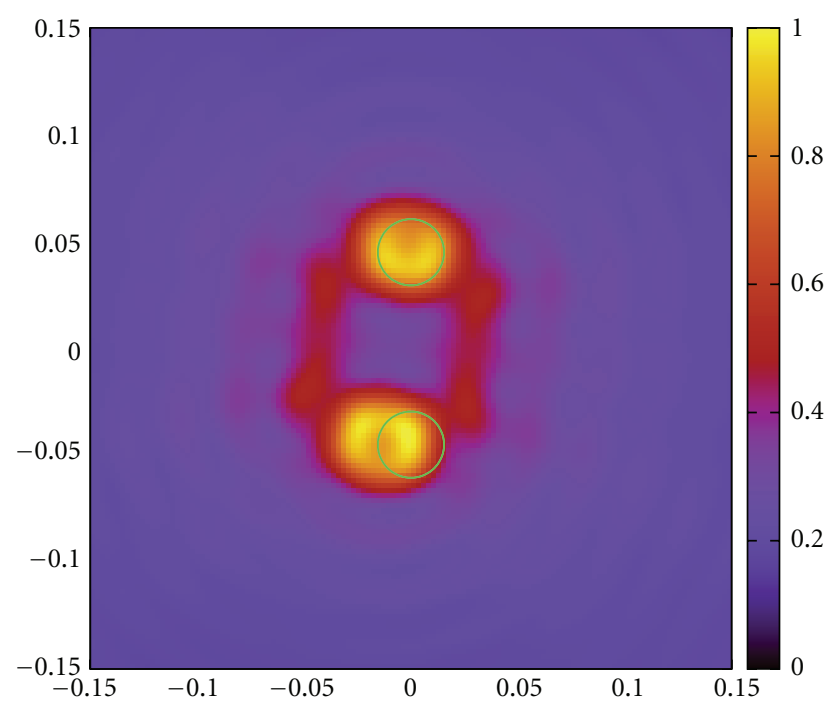

Figure 6: Reconstruction of a couple of twin dielectric cylinders. Multiview; map of the $P_{F}$ values computed at $f=8 \mathrm{GHz}$.

Multiview reconstructions can provide a much more regular result, with a limited background noise. The case of $f=8 \mathrm{GHz}$ is shown in Figure 6 .

Finally, in Figure 7, the result given by the multiviewmultifrequency method is shown. As it was in the case of a single cylinder, once again the combination of the results in frequency can provide a much better background.

3.3. Foam and Plastic Object. The third considered scatterer was the "FoamDielExt" object, included in the second Fresnel dataset [40]. The scatterer was made by two tangent cylinders with circular cross sections; one cylinder (radius $r_{1}=15.5 \mathrm{~mm}$ ) was plastic, with $\varepsilon_{r, 1}=3 \pm 0.3$, while the 


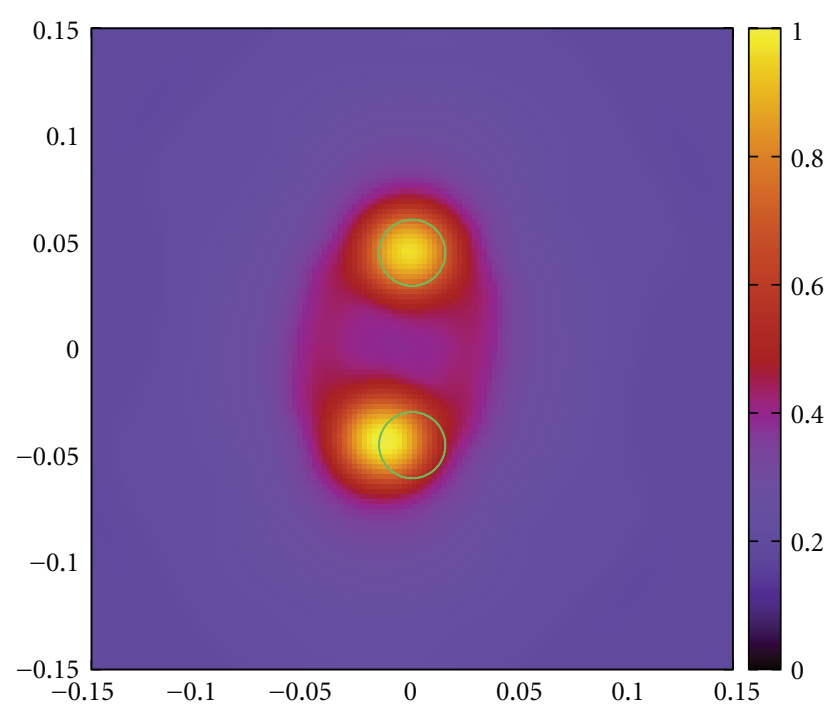

FIGURE 7: Reconstruction of a couple of twin dielectric cylinders. Multiview-multifrequency. Map of the $\mathcal{P}_{V F}$ values.

other one (radius $r_{2}=40 \mathrm{~mm}$ ) was made of a foam having $\varepsilon_{r, 2}=1.45 \pm 0.15$. The radius of the observation domain was $R=1.67 \mathrm{~m}$ and 240 samples were available at each frequency.

For this case, an accurate investigation about the right number of singular values was needed to achieve a good reconstruction. As an example, the limiting values of 8 and 210 singular values are considered, respectively, in Figures $8(\mathrm{a})$ and $8(\mathrm{~b})$. It can be seen that, by using a small number of singular values, the scattering from the foam cylinder is much more clear and sharp than in the case of 210 singular values. However, when only 8 singular values are used, information about the dielectric cylinder gets mostly lost and hence a trade-off is in order. Actually, depending on the frequency, a variable number of singular values, ranging from 35 to 350 were used for achieving the best results.

Another example of reconstruction is given in Figure 9, where the multiview map at $f=4 \mathrm{GHz}$ is shown. In this case, 140 singular values were used to achieve the better result. It can be seen that the different scattering given by the foam and by the plastic parts of the object can be clearly appreciated.

A further enhancement, with a pretty nice contrast between the two scattering cylinders, is achieved when results are combined also in frequency. The related map is shown in Figure 10.

3.4. Metallic Object. The metallic object was an U-shaped cylinder, having a cross-section with dimensions $80 \times$ $50 \mathrm{~mm}^{2}$, and the thickness of the metal was $5 \mathrm{~mm}$ [31]. Also in this case the analytical method is capable of recovering the presence of the target in the investigation domain, and a rectangular shape can be clearly recognized, when the various views are combined, especially at the higher frequencies.

Information provided by multifrequency can give a sharp reconstruction of a partial object, as it is shown in Figures 11 and 12 , but fail to give a reconstruction of the whole object.

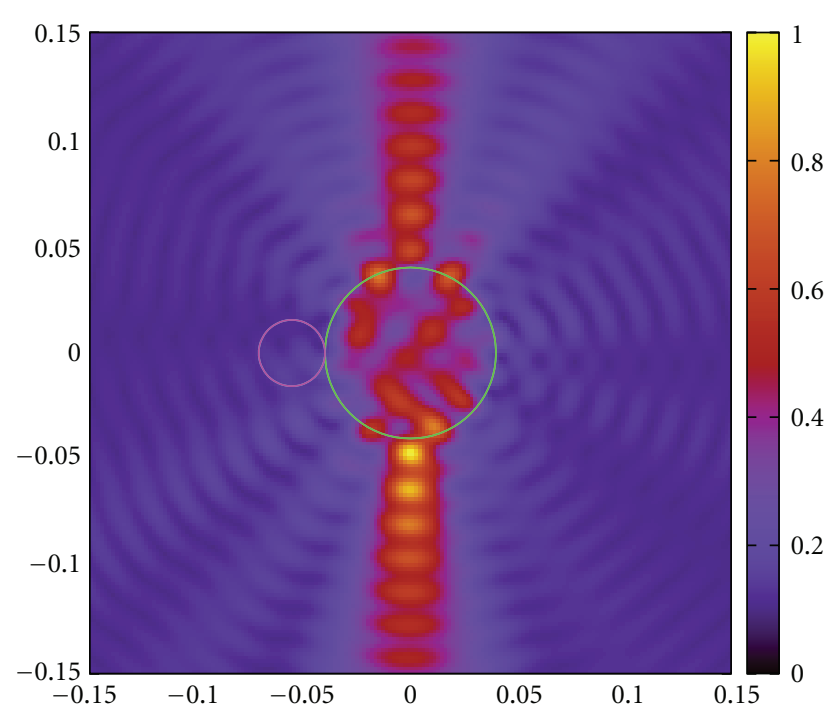

(a)

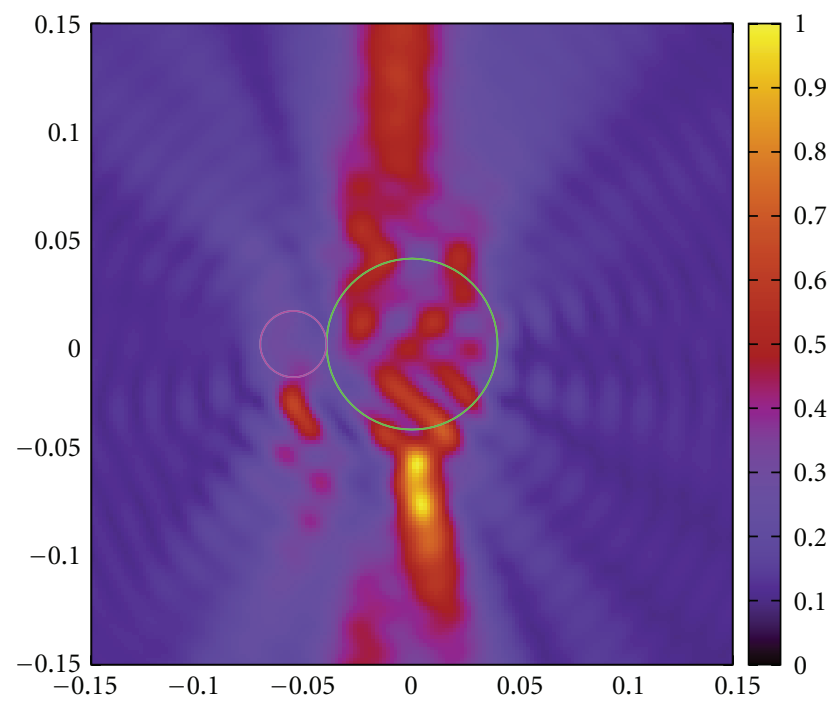

(b)

Figure 8: Reconstructions of the radiating source for the FoamDielExt object. Single view $\vartheta_{v}=90^{\circ}$ and single frequency $f=10 \mathrm{GHz}$. (a) 8 singular values were used. (b) 210 singular values were used.

As already mentioned, instead, multiview gives a much more better overall result. In particular, in Figure 13, the map of the multiview result, computed at $f=16 \mathrm{GHz}$, is shown. Although some resonances seem to appear inside the $\mathrm{U}$, it can be seen that the object can be recognized without any doubt, and it is worth noting that the open side is very different from the other sides.

As it was in the cases of dielectric objects, the multiviewmultifrequency approach can smooth the noise in the background. However, in this case, the results is not as good as it was for dielectric objects and the true object is smoothed, too. This result is shown in Figure 14. 


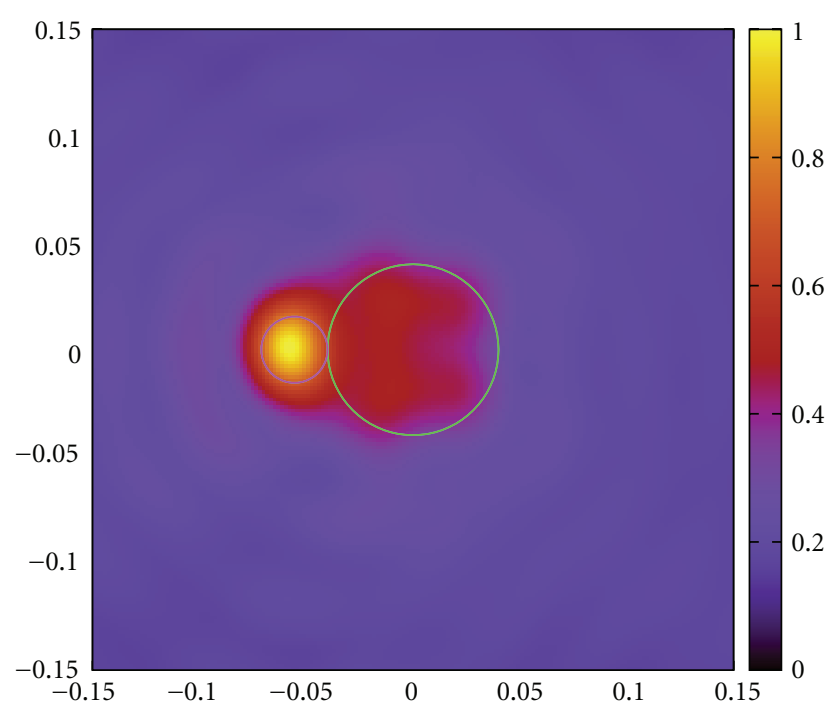

Figure 9: Reconstruction of a couple of the FoamDielExt object. Multiview; map of the $P_{F}$ values computed at $f=4 \mathrm{GHz}$. At this frequency 140 singular values were used.

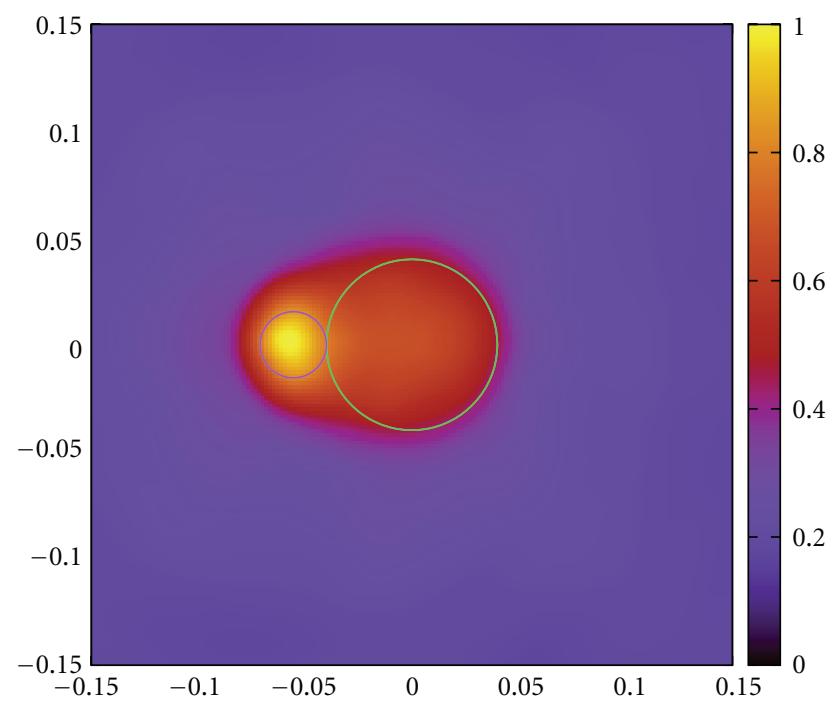

FIGURE 10: Reconstruction of a couple of twin dielectric cylinders. Multiview-multifrequency. Map of the $\mathcal{P}_{V F}$ values.

\section{Conclusions}

In this work, an analytical method for inverse scattering problems was used to successfully reconstruct both homogeneous and inhomogeneous targets from multifrequency multistatic experimental data. The proposed method uses the singular values decomposition of the scattering operator to achieve a closed solution to the integral equation of the electromagnetic scattering. The derivation of the radiating source density inside the domain of investigation is the first step of the work, as well as the preliminary result useful to locate a scattering object inside such a region.

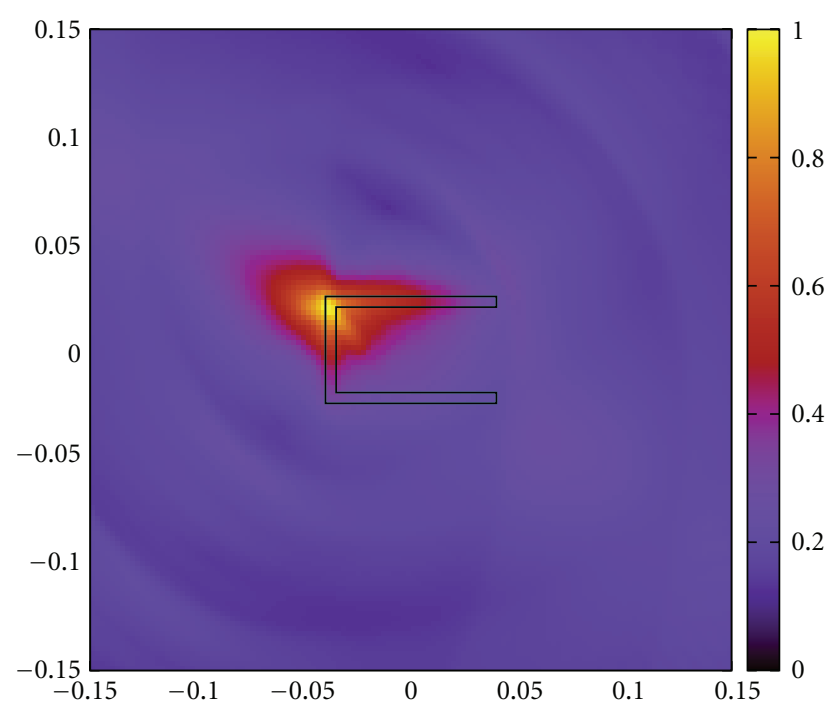

FIGURE 11: Reconstruction of a metallic U-shaped cylinder. Multifrequency; map of the $P_{V}$ values computed at $\vartheta_{v}=160^{\circ}$.

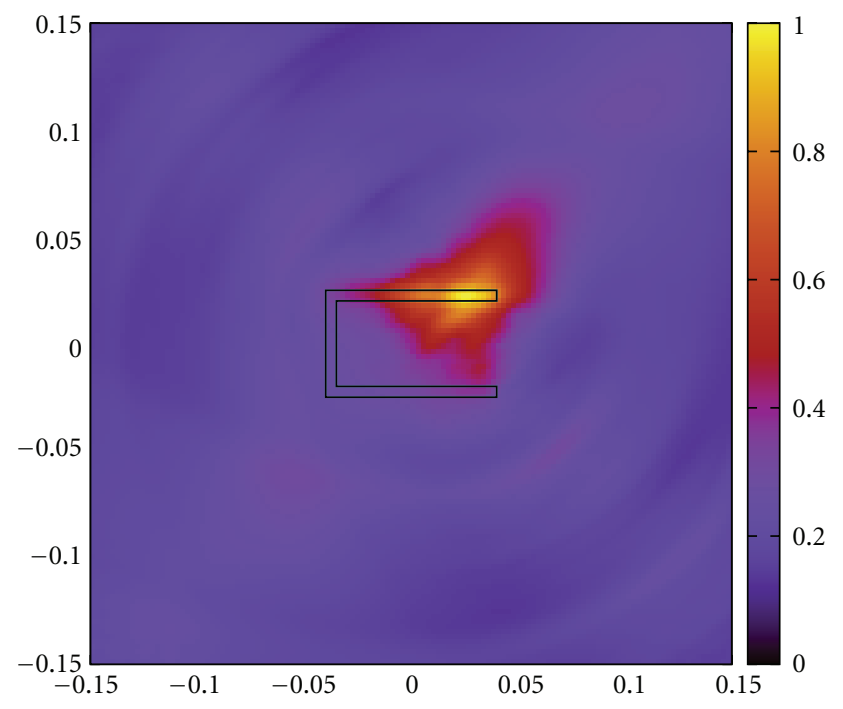

FIGURE 12: Reconstruction of a metallic U-shaped cylinder. Multifrequency; map of the $P_{V}$ values computed at $\vartheta_{v}=60^{\circ}$.

The dependence of the reconstructions on various parameters was investigated. In particular, results have shown that a correct choice of the significant number of singular values in the solution can lead to an optimal reconstruction, however; the method has proved robust enough to provide good reconstructions for a wide range of parameters.

Dielectric objects as well as objects made of conductive material were successfully tested.

As a general comment it should be stressed that the algorithm is very robust, and that, even for the very large domains used and with a suboptimal number of singular values, the object location was always clearly recovered. 


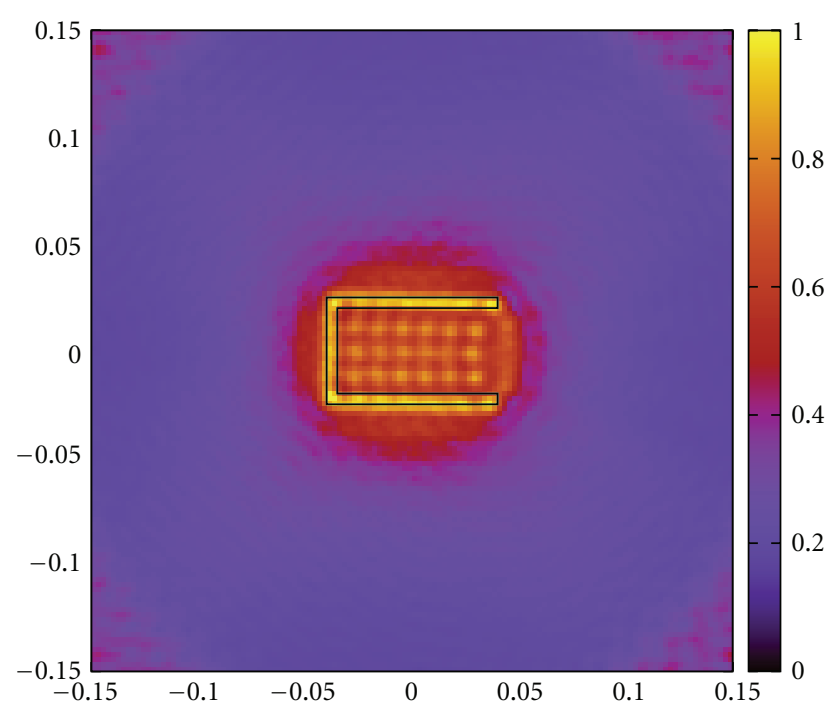

Figure 13: Reconstruction of a metallic U-shaped cylinder. Multiview; map of the $P_{F}$ values computed at $f=16 \mathrm{GHz}$.

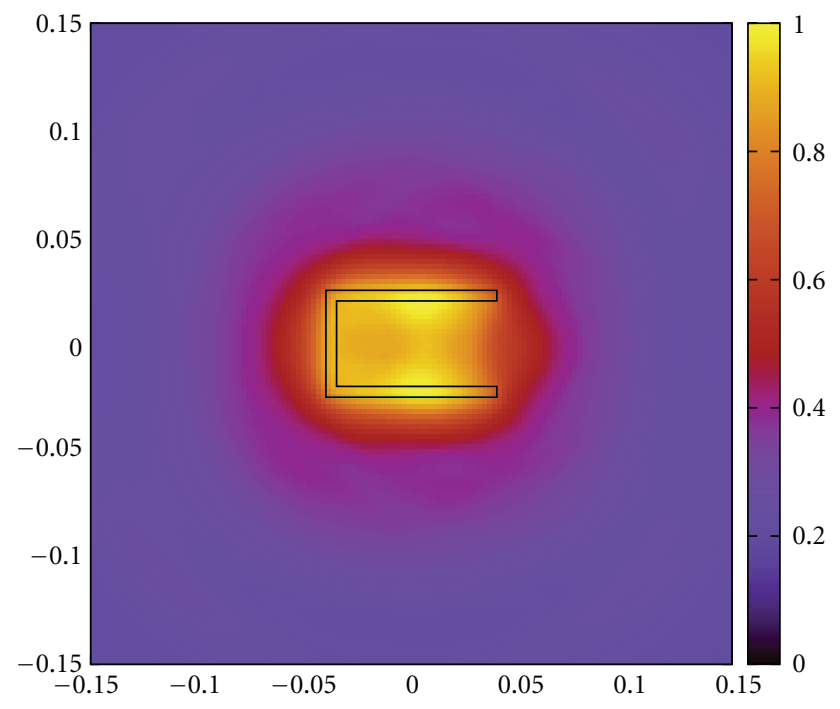

Figure 14: Reconstruction of the shape of a metallic U-shaped cylinder. Multiview-multifrequency. Map of the $\mathcal{P}_{V F}$ values.

Furthermore, thanks to the closed-form formulation of the solution, all the results were obtained in a very short computation time. It must also be pointed out, in particular, that, once the operating frequency and the dimensions of the investigation domain have been chosen, the singular system of the problem can be computed "offline." For the same reason also some other operations, for example, the inspection of the singular values, can be performed before the reconstruction process. As for the "online" part of the algorithm, also in this case things go very fast. As an extra bonus, since the closed-form virtually provides the solution at any point inside the investigation domain, high resolution maps of the reconstructed area can be obtained, without the constraints on grid dimensions and on the number of pixels usually imposed by algorithms based on numerical discretization of the problem.

Future work will be focused on the reconstruction of the scattering potential of the objects under test and on the possible use of a set of nonradiating sources to refine the results. It is expected that these steps can provide a notable enhancement in the results, as it was already proved by numerical simulations.

A further goal will be also testing the method on other experimental datasets, for example, on the series provided by the University of Manitoba [41].

\section{References}

[1] J. C. Bolomey and L. Jofre, "Three decades of active microwave imaging achievements, difficulties and future challenges," in Proceedings of the IEEE International Conference on Wireless Information Technology and Systems (ICWITS '10), pp. 1-4, Honolulu, Hawaii, USA, September 2010.

[2] M. Pastorino, Microwave Imaging, John Wiley \& Sons, New York, NY, USA, 2010.

[3] Y. J. Kim, L. Jofre, F. De Flaviis, and M. Q. Feng, "Microwave reflection tomographic array for damage detection of civil structures," IEEE Transactions on Antennas and Propagation, vol. 51, no. 11, pp. 3022-3032, 2003.

[4] K. J. Langenberg, K. Mayer, and R. Marklein, "Nondestructive testing of concrete with electromagnetic and elastic waves: modeling and imaging," Cement and Concrete Composites, vol. 28, no. 4, pp. 370-383, 2006.

[5] M. Benedetti, M. Donelli, A. Martini, M. Pastorino, A. Rosani, and A. Massa, "An innovative microwave-imaging technique for nondestructive evaluation: applications to civil structures monitoring and biological bodies inspection," IEEE Transactions on Instrumentation and Measurement, vol. 55, no. 6, pp. 1878-1884, 2006.

[6] N. Qaddoumi, R. Zoughi, and G. W. Carriveau, "Microwave detection and depth determination of disbonds in low-permittivity and low-loss thick sandwich composites," Re-earch in Nondestructive Evaluation, vol. 8, no. 1, pp. 51-63, 1996.

[7] S. Kharkovsky and R. Zoughi, "Microwave and millimeter wave nondestructive testing and evaluation," IEEE Instrumentation and Measurement Magazine, vol. 10, no. 2, pp. 26-38, 2007.

[8] R. Zoughi and S. Kharkovsky, "Microwave and millimetre wave sensors for crack detection," Fatigue and Fracture of Engineering Materials and Structures, vol. 31, no. 8, pp. 695713, 2008.

[9] Y. Deng and X. Liu, "Electromagnetic imaging methods for nondestructive evaluation applications," Sensors, vol. 11, pp. 11774-11808, 2011.

[10] M. Pastorino, "Recent inversion procedures for microwave imaging in biomedical, subsurface detection and nondestructive evaluation applications," Measurement, vol. 36, no. 3-4, pp. 257-269, 2004.

[11] M. Bertero and M. Piana, "Inverse problems in biomedical imaging: modeling and methods of solution," in Complex Systems in Biomedicine, A. Quarteroni, L. Formaggia, and A. Veneziani, Eds., pp. 1-33, Springer, Milan, Italy, 2006.

[12] S. C. Hagness, "Microwave imaging in medicine: promises and future challenges," in Proceedings of the URSI General Assembly, pp. 7-16, Chicago, Ill, USA, August 2008. 
[13] S. Semenov, "Microwave tomography: review of the progress towards clinical applications," Philosophical Transactions of the Royal Society A, vol. 367, no. 1900, pp. 3021-3042, 2009.

[14] P. M. Meaney and K. D. Paulsen, "Challenges on microwave imaging supported by clinical results," in Proceedings of the International Workshop on Biological Effects of Electromagnetic Fields, pp. 10-14, Bodrum, Turkey, October 2010.

[15] A. Salvadè, M. Pastorino, R. Monleone et al., "Microwave imaging of foreign bodies inside wood trunks," in Proceedings of the IEEE Workshop on Imaging Systems and Techniques (IST '08), pp. 88-93, Chania, Greece, September 2008.

[16] H. Park, C. C. Chang, B. Deng et al., "Recent advancements in microwave imaging plasma diagnostics," Review of Scientific Instruments, vol. 74, pp. 4239-4262, 2003.

[17] A. Alu and N. Engheta, "Plasmonic and metamaterial cloaking: physical mechanisms and potentials," Journal of Optics A, vol. 10, no. 9, Article ID 093002, 2008.

[18] S. Caorsi, A. Costa, and M. Pastorino, "Microwave imaging within the second-order born approximation: stochastic optimization by a genetic algorithm," IEEE Transactions on Antennas and Propagation, vol. 49, no. 1, pp. 22-31, 2001.

[19] M. Benedetti, M. Donelli, G. Franceschini, M. Pastorino, and A. Massa, "Effective exploitation of the a priori information through a microwave imaging procedure based on the SMW for NDE/NDT applications," IEEE Transactions on Geoscience and Remote Sensing, vol. 43, no. 11, pp. 2584-2592, 2005.

[20] G. Bozza, C. Estatico, M. Pastorino, and A. Randazzo, "Application of an inexact-Newton method within the secondorder Born approximation to buried objects," IEEE Geoscience and Remote Sensing Letters, vol. 4, no. 1, pp. 51-55, 2007.

[21] G. Bozza, C. Estatico, A. Massa, M. Pastorino, and A. Randazzo, "Short-range image-based method for the inspection of strong scatterers using microwaves," IEEE Transactions on Instrumentation and Measurement, vol. 56, no. 4, pp. 11811188, 2007.

[22] A. Randazzoa and C. Estatico, "A regularisation scheme for electromagnetic inverse problems: application to crack detection in civil structures," Nondestructive Testing and Evaluation. In press.

[23] B. Hofmann, On Ill-Posedness and Local Ill-Posedness of Operator Equations in Hilbert Spaces, Chemnitz University of Technology, 1997.

[24] A. J. Devaney and G. C. Sherman, "Nonuniqueness in inverse source and scattering problems," IEEE Transactions on Antennas and Propagation, vol. 30, pp. 1034-1042, 1982.

[25] M. Chiappe and G. L. Gragnani, "An analytical approach to the reconstruction of the radiating currents in inverse electromagnetic scattering," Microwave and Optical Technology Letters, vol. 49, no. 2, pp. 354-360, 2007.

[26] G. L. Gragnani, "Shape reconstruction of 2-D dielectric objects by an analytical method," International Journal of Signal and Imaging Systems Engineering, vol. 3, no. 2, pp. 8192, 2010.

[27] R. V. McGahan and R. E. Kleinman, "Special session on image reconstruction using real data," IEEE Antennas and Propagation Magazine, vol. 38, no. 3, pp. 39-40, 1996.

[28] R. V. McGahan and R. E. Kleinman, "Second annual special session on image reconstruction using real data," IEEE Antennas and Propagation Magazine, vol. 39, no. 2, pp. 7-9, 1997.

[29] R. V. McGahan and R. E. Kleinman, "The third annual special session on image reconstruction using real data, Part 1," IEEE Antennas and Propagation Magazine, vol. 41, no. 1, pp. 34-51, 1999.
[30] R. V. McGahan and R. E. Kleinman, "The third annual special session on image reconstruction using real data Part 2," IEEE Antennas and Propagation Magazine, vol. 41, no. 1, pp. 20-40, 1999.

[31] K. Belkebir and M. Saillard, "Special section: testing inversion algorithms against experimental data," Inverse Problems, vol. 17, no. 6, pp. 1565-1571, 2001.

[32] K. Belkebir and M. Saillard, "Testing inversion algorithms against experimental data: inhomogeneous targets," Inverse Problems, vol. 21, pp. S1-S3, 2005.

[33] C. A. Balanis, Advanced Engineering Electromagnetics, John Wiley \& Sons, New York, NY, USA, 1989.

[34] D. E. Livesay and K. M. Chen, "Electromagnetic field induced inside arbitrarily shaped biological bodies," IEEE Transactions on Microwave Theory and Techniques, vol. 22, no. 12, pp. 127380, 1974.

[35] S. Caorsi, A. Frattoni, G. L. Gragnani, E. Nortino, and M. Pastorino, "Numerical algorithm for dielectric-permittivity microwave imaging of inhomogeneous biological bodies," Medical and Biological Engineering and Computing, vol. 29, no. 6, pp. NS37-NS38, 1991.

[36] D. G. Dudley, T. M. Habashy, and E. Wolf, "Linear inverse problems in wave motion: nonsymmetric first-kind integral equations," IEEE Transactions on Antennas and Propagation, vol. 48, no. 10, pp. 1607-1617, 2000.

[37] A. Gamliel, K. Kim, A. I. Nachman, and E. Wolf, "A new method for specifying nonradiating, monochromatic, scalar sources and their fields," Journal of the Optical Society of America, vol. 6, no. 9, pp. 1388-1393, 1989.

[38] E. Wallacher and A. K. Louis, "Complete sets of radiating and nonradiating parts of a source and their fields with applications in inverse scattering limited-angle problems," International Journal of Biomedical Imaging, vol. 2006, Article ID 93074, 2006.

[39] G. L. Gragnani and M. DiazMendez, "Improved electromagnetic inverse scattering procedure using non-radiating sources and scattering support reconstruction," IET Microwaves Antennas \& Propagation, vol. 5, no. 15, pp. 1822-1829, 2011.

[40] J.-M. Geffrin, P. Sabouroux, and C. Eyraud, "Free space experimental scattering database continuation: experimental set-up and measurement precision," Inverse Problems, vol. 21, no. 6, pp. S117-S130, 2005.

[41] C. Gilmore, A. Zakaria, P. Mojabi, M. Ostadrahimi, S. Pistorius, and J. LoVetri, "The University of ManitobaMicrowave Imaging Repository: a two-dimensionalmicrowave scattering database for testing inversion and calibration algorithms," IEEE Antennas and Propagation Magazine, vol. 53, no. 5, pp. 126-133, 2011. 

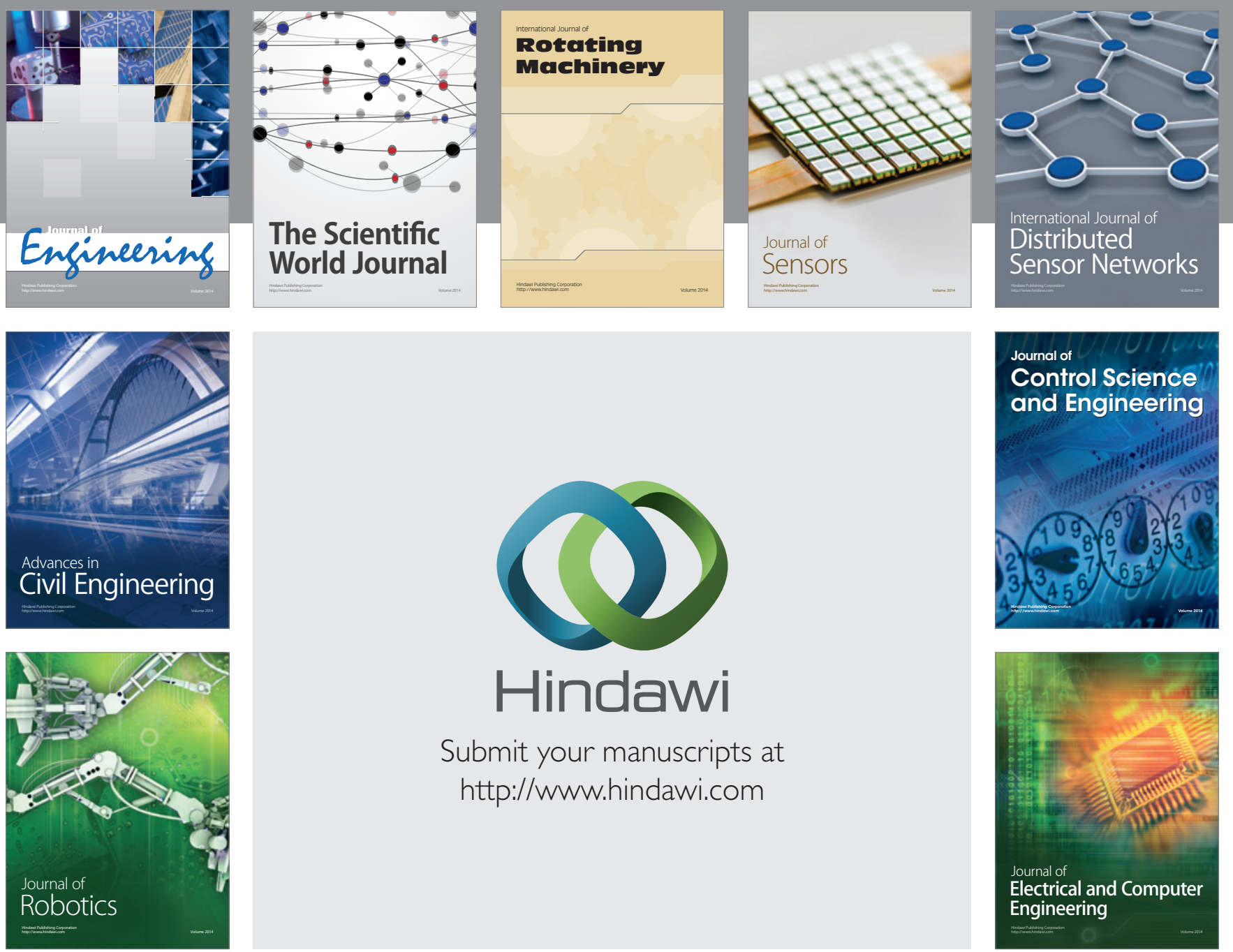

Submit your manuscripts at

http://www.hindawi.com
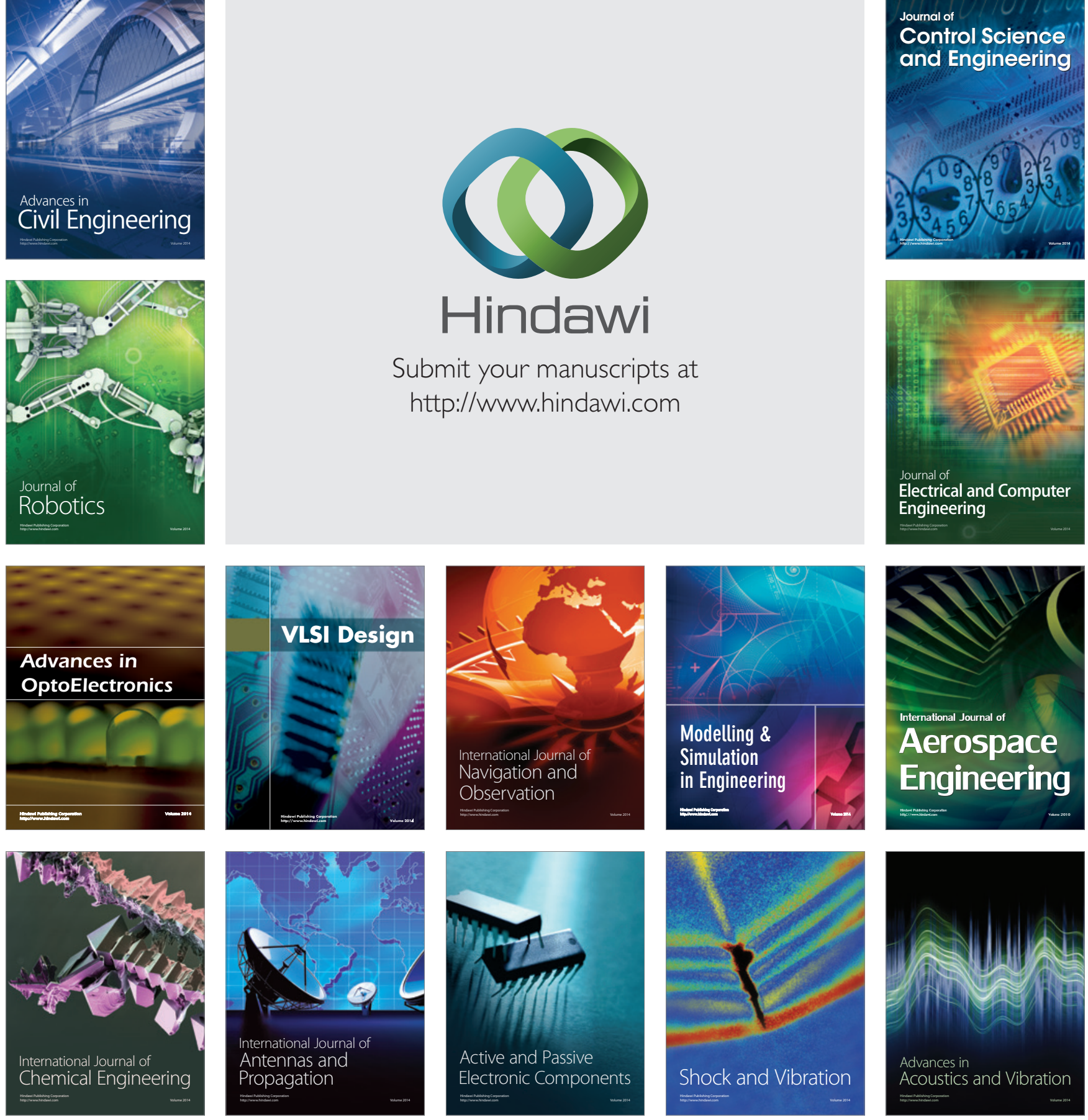